\title{
Analysis of vertebral augmentation practice patterns: a 2016 update
}

\author{
Joshua A Hirsch, ${ }^{1}$ Ronil V Chandra, ${ }^{2}$ Vidsysagar Pampati, ${ }^{3}$ John D Barr, ${ }^{4}$ \\ Allan L Brook, ${ }^{5}$ Laxmaiah Manchikanti ${ }^{6}$
}

${ }^{1}$ Neuroendovascular Program and Neurolnterventional Spine Service, Massachusetts General Hospital, Harvard Medical School, Boston, Massachusetts, USA

${ }^{2}$ Interventional Neuroradiology Service, Departments of Medicine and Surgery, Monash Imaging, Monash Medical Centre, Monash University, Melbourne, Victoria, Australia ${ }^{3}$ Pain Management Center of Paducah, University of Louisville, Paducah, Kentucky, USA

${ }^{4}$ Departments of Radiology and Neurological Surgery, UT Southwestern Medical Center, Dallas, Texas, USA ${ }^{5}$ Montefiore Medical Center, Albert Einstein College of Medicine, Bronx, New York, USA

${ }^{6}$ Pain Management Center of Paducah, University of Louisville, Louisville, Kentucky, USA

\section{Correspondence to}

Dr J A Hirsch,

Neuroendovascular Program and Neurolnterventional Spine Service, Massachusetts General Hospital, Harvard Medical School, Boston, MA 02114, USA; Hirsch@snisonline.org

Accepted 20 September 2016

\section{CrossMark}

To cite: Hirsch JA,

Chandra RV, Pampati V,

et al. I Neurolntervent Surg

2016;8:1299-1304.

\section{ABSTRACT}

Objective To evaluate procedure utilization patterns for vertebroplasty and kyphoplasty in the US Medicare

population from 2004 to 2014.

Methods The analysis was performed using the Centers for Medicare and Medicaid Services database of specialty utilization files for the fee for service (FFS) Medicare population.

Results The FFS Medicare population increased by $28 \%$ with an annual increase of $2.5 \%$ from 2004 to

2014. Utilization of vertebroplasty procedures decreased by $63 \%$ with an average annual decrease of $9.5 \%$ from 2004 to 2014 per 100000 FFS Medicare beneficiaries. During the same time period, kyphoplasty procedures decreased by a total of $10 \%$, with an average annual decrease of $1.3 \%$. For augmentation generally (combined vertebroplasty/kyphoplasty data) there was thus an overall decrease in the rate per 100000 Medicare population of 32\% from 2004 to 2014, with an average annual decrease of $4.8 \%$. The majority of vertebroplasty procedures were performed by radiologists whereas the majority of kyphoplasties were performed by orthopedic surgeons and neurosurgeons.

Conclusions There has been a significant decline in vertebroplasty and kyphoplasty procedures in the FFS Medicare population between 2004 and 2014.

\section{INTRODUCTION}

Vertebral compression fractures are the most common type of osteoporotic fracture and are also associated with malignancy. Osteoporotic vertebral fractures affect 117 per 100000 persons, with the primary symptom of back pain that can be debilitating. ${ }^{1} 2$ These fractures are associated with a prolonged impact on health related quality of life, ${ }^{2}$ and the direct management costs are estimated at more than $\$ 1$ billion per annum. ${ }^{3}$ Similarly, patients with malignancy commonly develop bone metastases. Vertebral fractures in cancer patients may be due to metastases, osteonecrosis after radiation therapy, and/or osteopenia caused by systemic anticancer treatments.

In these cohorts of patients, minimally invasive percutaneous treatments, such as vertebroplasty and kyphoplasty, have become common in the USA. Initial enthusiasm was driven by multiple observational studies and comprehensive reviews showing positive results. $^{4-7}$ In fact, vertebroplasty increased from a rate of 43 per 100000 Medicare population in 2001 to 85 in $2008 .^{8}$ Along the same lines, kyphoplasty increased from 120 per 100000 Medicare population to 141 in 2008. However, two randomized controlled trials of vertebroplasty for osteoporotic spinal fractures were published in the New England Journal of Medicine (NEJM) in 2009..$^{10}$ These trials showed lack of effectiveness of vertebroplasty compared with sham surgery, and impacted on the utilization rates of both vertebroplasty and kyphoplasty. While utilization patterns of other interventional techniques continued to demonstrate substantial increases, ${ }^{11-13}$ vertebroplasty decreased from 85 per 100000 Medicare population to 49 in 2010, and kyphoplasty decreased from 141 per 100000 Medicare population to 121 in the year $2010 .^{8}$

Since publication of these two trials in 2009 , multiple additional randomized controlled trials confirmed prior observations that have demonstrated benefit for vertebroplasty and kyphoplasty for both osteoporotic and cancer related vertebral fractures compared with conservative care. ${ }^{14-20}$ Recently, vertebroplasty has also been demonstrated to be effective compared with sham surgery for patients with acute fractures. ${ }^{21}$ Thus our aim was to evaluate the procedure utilization patterns for vertebroplasty and kyphoplasty in the Medicare population from 2004 to 2014.

\section{MATERIALS AND METHODS}

This analysis of data of utilization patterns of vertebroplasty and kyphoplasty procedures was performed following the reporting standards of Strengthening the Reporting of Observational Studies in Epidemiology (STROBE) guidance. ${ }^{22}$ Institutional review board approval was not required for this assessment as public use files available through the Centers for Medicare and Medicaid Services (CMS) database was utilized. ${ }^{23}$ This analysis exclusively used non-attributable de-identified data.

\section{Study design}

The purpose of the study was to retrospectively evaluate the utilization patterns of thoracolumbar vertebroplasty and kyphoplasty procedures from 2004 to 2014 by fee for service (FFS) Medicare beneficiaries. These physician/supplier procedure summary master files were aggregates of all Medicare part B billing claims for services performed in the USA by all providers. These data were purchased from the CMS.

\section{Setting}

The CMS database of specialty utilization from 2004 to 2014 data files of FFS Medicare was utilized. $^{23}$ This is a $100 \%$ sample. 


\section{Measures}

Services were defined as submitted, allowed, denied, and those with zero payments. Allowed services constituted total services after deletion of denied services and services with zero payments. For each procedure, total allowed services and rates per 100000 were calculated for the corresponding year.

\section{Current procedural terminology codes assessed}

For this study of utilization patterns, the current procedural terminology procedure codes were used for vertebroplasty procedures (22 520 - thoracic percutaneous vertebroplasty; 22 521lumbar vertebroplasty; 22 522-vertebroplasty, each additional thoracic or lumbar vertebral body) and kyphoplasty procedures (22 523-thoracic kyphoplasty; 22 524-lumbar kyphoplasty; 22 525-kyphoplasty, each additional thoracic or lumbar vertebral body). Vertebroplasty and kyphoplasty procedures were approved by CMS in January 2001 and 2006, respectively.

Vertebroplasty and kyphoplasty procedures were performed historically by various types of providers, even though the majority are performed by specialists representing radiology (diagnostic-30 and interventional radiology-94), orthopedic surgery (20), neurosurgery, pain management group (interventional pain management-09 or pain management-72), anesthesiology (05), and physical medicine and rehabilitation (25), and the remaining specialties grouped as other physicians. Site of service was also analyzed and categorized as hospital outpatient departments, hospital inpatient, ambulatory surgery centers (ASCs), and in physicians' offices (in office).

\section{Statistical analysis}

The data were analyzed using Microsoft Access 2003 and Microsoft Excel 2003 (Microsoft, Redmond, Washington, USA); procedure utilization was calculated per 100000 Medicare beneficiaries.

\section{RESULTS}

\section{Population characteristics}

Table 1 shows the growth of the US population and FFS Medicare beneficiaries along with overall utilization patterns of 312678 vertebroplasty and kyphoplasty procedures from 2004 to 2014, and the growth rate per 100000 FFS Medicare beneficiaries over the decade. Overall, the US population increased by $9 \%$ whereas the population over 65 years of age increased by $27 \%$ with an annual increase of $2.4 \%$ from 2004 to 2014 . The elderly population over 65 years of age constituted $12.4 \%$ of the population in 2004 increasing to $14.5 \%$ in 2014. FFS Medicare beneficiaries, including those $>65$ years of age and also disabled individuals less than 65 years of age, increased to $28 \%$ of the US population from 2004 to 2014. Overall utilization of vertebroplasty procedures decreased by $63 \%$, with an annual decrease of $9.5 \%$ from 2004 to 2014 per 100000 FFS Medicare beneficiaries, whereas kyphoplasty procedures decreased by $10 \%$, with an annual decrease of $1.3 \%$ from 2006 to 2014 per 100000 FFS Medicare beneficiaries.

\section{Utilization characteristics}

Table 2 show a summary of the frequency of utilization of vertebroplasty and kyphoplasty procedures in fee for service (FFS) Medicare beneficiaries from 2006 to 2014 with distribution of procedural characteristics.

As shown in table 2, the overall rate of decrease for vertebroplasty and kyphoplasty combined procedures was 32\%, with an annual decrease of $4.8 \%$ per 100000 FFS Medicare beneficiaries from 2006 to 2014; however, vertebroplasty procedures decreased by $67 \%$, with an annual decrease of $12.8 \%$ per 100000 FFS Medicare population from 2006 to 2014. In contrast, kyphoplasty procedures increased from 2006 to 2009, with a slight decrease starting in 2010 , an overall decrease of $10 \%$, and an annual decrease of $1.3 \%$ per 100000 Medicare population, starting with 126 per 100000 Medicare population and declining to 113 . However, vertebroplasty procedures were 82 per 100000 Medicare population in 2006 and declined to 28 in 2014. Initially there appeared to be a slight shift of procedures from vertebroplasty to kyphoplasty with a stunted growth until the publication of two randomized controlled trials ${ }^{9}{ }^{10}$ following which the decline was rapid. ${ }^{8} 2425$ After publication of the randomized trials, ${ }^{9} 10$ kyphoplasty also plateaued, with some decreases from 138 per 100000 Medicare population in 2009, to 123 in 2010, 122 in 2011 and 2012, 119 in 2013, and finally, 113 in 2014.

\section{Specialty characteristics}

As shown in table 3, 75.3\% of vertebroplasty procedures were performed by radiologists whereas $71.2 \%$ of kyphoplasty procedures were performed by orthopedic surgeons and neurosurgeons in 2006. There was a slight increase in utilization of kyphoplasty procedures for anesthesiology and pain management specialties from 2010 when these procedures were approved for use in ASCs, making reimbursement in this site of service possible for CMS patients. Specialty designation derives from the electronic portal through which physicians enroll in Medicare, the so called Provider Enrollment and Chain/ Ownership System (PECOS), leading to significant confusionfor example, many interventional radiologists are listed as diagnostic. The same is true for interventional pain management and pain management. For that reason, we combined diagnostic and interventional radiologists in the specialty specific analysis.

Vertebroplasty utilization decreased by $2 \%$ from 2006 to 2009, and decreased by $18 \%$ from 2009 to 2010, and continued to decrease by $49 \%$ from 2010 to 2014 . Kyphoplasty procedure utilization increased by $15 \%$ from 2006 to 2009 , decreased by $8 \%$ from 2009 to 2010 , and increased by $5 \%$ from 2010 to 2014 (table 4).

\section{Site of service utilization}

Place of service data were available from 2008. The majority of vertebroplasty and kyphoplasty procedures (>90\%) were performed in hospitals, as either outpatient (53\%) or inpatient (33\%) procedures. There was a slight increase in ASC settings from $1.4 \%$ in 2009 to $3.6 \%$ in 2014 , as shown in table 5 .

\section{Discussion}

The present analysis shows significant change in utilization patterns of vertebral augmentation procedures over the past decade. There were substantial decreases in vertebroplasty procedures of $63 \%$ from 2004 to 2014 and a modest decrease of $10 \%$ for kyphoplasty procedures from 2006 to 2014. After the NEJM publications in 2009 , vertebroplasty decreased by $49 \%$ from 2010 to 2014, while kyphoplasty utilization increased by $5 \%$. This overall reduction in vertebral augmentation procedures occurred in spite of more recent positive randomized controlled trials. This suggests that the 2009 NEJM publications have had considerable ongoing impact on vertebral augmentation utilization rates in the FFS Medicare population. 
Table 1 Summary of the growth of the US population fee for service Medicare beneficiaries and frequency of utilization of vertebroplasty and kyphoplasty procedures in the Medicare population from 2004 to 2014

\begin{tabular}{|c|c|c|c|c|c|c|c|c|c|c|}
\hline \multirow[b]{2}{*}{ Year } & \multirow[b]{2}{*}{$\begin{array}{l}\text { US total } \\
\text { population in } \\
\text { thousands ( } \% \\
\text { elderly) }\end{array}$} & \multirow[b]{2}{*}{$\begin{array}{l}\text { Medicare } \\
\text { beneficiaries in } \\
\text { thousands (\% } \\
\text { elderly) }\end{array}$} & \multicolumn{4}{|c|}{ Vertebroplasty } & \multicolumn{4}{|c|}{ Kyphoplasty } \\
\hline & & & Services & $\begin{array}{l}\% \text { Change } \\
\text { from } \\
\text { previous year }\end{array}$ & Rate* & $\begin{array}{l}\% \text { Change } \\
\text { from } \\
\text { previous year }\end{array}$ & Services & $\begin{array}{l}\% \text { Change } \\
\text { from } \\
\text { previous year }\end{array}$ & Rate* & $\begin{array}{l}\% \text { Change } \\
\text { from } \\
\text { previous year }\end{array}$ \\
\hline 2004 & $292892(12.4)$ & $41729(84.7)$ & 31032 & & 74 & - & - & - & - & - \\
\hline 2005 & 295561 (12.4) & $42496(84.2)$ & 35478 & 14 & 83 & 12.2 & - & - & - & - \\
\hline 2006 & $299395(12.4)$ & 43339 (83.8) & 35716 & 1 & 82 & -1.2 & 54657 & - & 126 & - \\
\hline 2007 & 301290 (12.6) & $44263(83.5)$ & 35175 & -2 & 79 & -3.7 & 61785 & 13 & 140 & 13.0 \\
\hline 2008 & $304056(12.8)$ & 45412 (83.4) & 36745 & 4 & 81 & 2.5 & 63348 & 3 & 139 & 2.5 \\
\hline 2009 & $307006(12.9)$ & 45801 (83.4) & 35091 & -5 & 77 & -4.9 & 63005 & -1 & 138 & -0.5 \\
\hline 2010 & $308746(13.0)$ & 46914 (83.1) & 28949 & -18 & 62 & -19.5 & 57890 & -8 & 123 & -8.1 \\
\hline 2011 & $311583(13.3)$ & $48300(82.8)$ & 24315 & -16 & 50 & -19.4 & 59043 & 2 & 122 & 2.0 \\
\hline 2012 & $313874(13.8)$ & $50300(83.3)$ & 18764 & -23 & 37 & -26.0 & 61165 & 4 & 122 & 3.6 \\
\hline 2013 & $316129(14.1)$ & $51900(83.0)$ & 16681 & -11 & 32 & -13.5 & 61839 & 1 & 119 & 1.1 \\
\hline 2014 & 318892 (14.5) & $53500(83.4)$ & 14732 & -12 & 28 & -12.5 & 60656 & -2 & 113 & -1.9 \\
\hline $\begin{array}{l}\text { Change } \\
(\%)\end{array}$ & 9 & 28 & -53 & & -63 & & 11 & & -10 & \\
\hline GGR (\%) & 0.9 & 2.5 & -7.2 & & -9.5 & & 1.3 & & -1.3 & \\
\hline
\end{tabular}

*Rate, per 100000 fee for service Medicare beneficiaries; kyphoplasty data available from 2006.

GGR, geometric growth rate.

Table 2 Utilization of vertebroplasty and kyphoplasty services in Medicare beneficiaries

\begin{tabular}{|c|c|c|c|c|c|c|c|c|c|c|c|c|}
\hline & $\begin{array}{l}\text { Current procedural } \\
\text { terminology }\end{array}$ & F2006 & F2007 & F2008 & F2009 & F2010 & F2011 & F2012 & F2013 & F2014 & Change (\%) & GGR (\%) \\
\hline \multirow{6}{*}{ Vertebroplasty } & Medicare & 43339 & 44263 & 45412 & 45801 & 46914 & 48300 & 50300 & 51900 & 53500 & 23 & 2.7 \\
\hline & Change (\%) & 2 & 2 & 3 & 1 & 2 & 3 & 4 & 3 & 3 & & \\
\hline & 22520 & 14435 & 14032 & 14559 & 13845 & 11430 & 9768 & 7469 & 6725 & 5842 & -60 & -10.7 \\
\hline & Change (\%) & -1 & -3 & 4 & -5 & -17 & -15 & -24 & -10 & -13 & & \\
\hline & Rate & 33 & 32 & 32 & 30 & 24 & 20 & 15 & 13 & 11 & -67 & -13.0 \\
\hline & 22521 & 14187 & 14491 & 15036 & 14458 & 12101 & 10275 & 7709 & 6812 & 6058 & -57 & -10.1 \\
\hline \multirow{17}{*}{ Kyphoplasty } & Change (\%) & 2 & 2 & 4 & -4 & -16 & -15 & -25 & -12 & -11 & & \\
\hline & Rate & 33 & 33 & 33 & 32 & 26 & 21 & 15 & 13 & 11 & -65 & -12.4 \\
\hline & 22522 & 7094 & 6652 & 7150 & 6788 & 5418 & 4272 & 3586 & 3144 & 2832 & -60 & -10.8 \\
\hline & Change (\%) & 2 & -6 & 7 & -5 & -20 & -21 & -16 & -12 & -10 & & \\
\hline & Rate & 16 & 15 & 16 & 15 & 12 & 9 & 7 & 6 & 5 & -68 & -13.2 \\
\hline & Vertebroplasty & 35716 & 35175 & 36745 & 35091 & 28949 & 24315 & 18764 & 16681 & 14732 & -59 & -10.5 \\
\hline & Change (\%) & 1 & -2 & 4 & -5 & -18 & -16 & -23 & -11 & -12 & & \\
\hline & Rate & 82 & 79 & 81 & 77 & 62 & 50 & 37 & 32 & 28 & -67 & -12.8 \\
\hline & 22523 & 21096 & 23690 & 23960 & 23550 & 21680 & 22044 & 22662 & 22921 & 22258 & 6 & 0.7 \\
\hline & Change (\%) & & 12 & 1 & -2 & -8 & 2 & 3 & 1 & -3 & & \\
\hline & Rate & 49 & 54 & 53 & 51 & 46 & 46 & 45 & 44 & 42 & -15 & -1.9 \\
\hline & 22524 & 22111 & 25220 & 26300 & 26305 & 24199 & 24699 & 26130 & 26646 & 26266 & 19 & 2.2 \\
\hline & Change (\%) & & 14 & 4 & 0 & -8 & 2 & 6 & 2 & -1 & & \\
\hline & Rate & 51 & 57 & 58 & 57 & 52 & 51 & 52 & 51 & 49 & -4 & -0.5 \\
\hline & 22525 & 11450 & 12875 & 13088 & 13150 & 12011 & 12300 & 12373 & 12272 & 12132 & 6 & 0.7 \\
\hline & Change (\%) & & 12 & 2 & 0 & -9 & 2 & 1 & -1 & -1 & & \\
\hline & Rate & 26 & 29 & 29 & 29 & 26 & 25 & 25 & 24 & 23 & -14 & -1.9 \\
\hline \multirow{6}{*}{ Combined } & Kyphoplasty & 54657 & 61785 & 63348 & 63005 & 57890 & 59043 & 61165 & 61839 & 60656 & 11 & 1.3 \\
\hline & Change (\%) & & 13 & 3 & -1 & -8 & 2 & 4 & 1 & -2 & & \\
\hline & Rate & 126 & 140 & 139 & 138 & 123 & 122 & 122 & 119 & 113 & -10 & -1.3 \\
\hline & $\begin{array}{l}\text { Vertebroplasty and } \\
\text { kyphoplasty }\end{array}$ & 90373 & 96960 & 100093 & 98096 & 86839 & 83358 & 79929 & 78520 & 75388 & -17 & -2.2 \\
\hline & Change (\%) & NA & 7 & 3 & -2 & -11 & -4 & -4 & -2 & -4 & & \\
\hline & Rate & 209 & 219 & 220 & 214 & 185 & 173 & 159 & 151 & 141 & -32 & -4.8 \\
\hline
\end{tabular}

Notably, the significant in reduction in vertebroplasty rates from 2010 to 2014 contrasts with the slight increase in kyphoplasty rates during the same period. This likely relates to multiple factors, most importantly the lack of benefit from vertebroplasty reported in the two NEJM trials (78 and 131 patients). ${ }^{9} 10$ The simultaneous publication in a very high impact factor journal (NEJM impact factor 47.1 in 2009) ${ }^{26}$ garnered significant media attention, and thus the results were widely known by both potential referring physicians and patients. This contrasts with an earlier trial of kyphoplasty compared with conservative 
Table 3 Utilization of vertebroplasty and kyphoplasty services by provider specialty in fee for service Medicare beneficiaries

\begin{tabular}{|c|c|c|c|c|c|c|c|c|c|c|c|}
\hline & F2006 & F2007 & F2008 & F2009 & F2010 & F2011 & F2012 & F2013 & F2014 & Change (\%) & GGR (\%) \\
\hline \multicolumn{12}{|l|}{ Vertebroplasty (n (\%)) } \\
\hline Radiology & $26909(75.3)$ & $25843(73.5)$ & $25786(70.2)$ & $24114(68.7)$ & $20546(71.0)$ & $17717(72.9)$ & $14162(75.5)$ & $12837(77.0)$ & $11391(77.3)$ & -58 & -10.2 \\
\hline Orthopedic surgery & $2914(8.2)$ & $3106(8.8)$ & $3763(10.2)$ & $3672(10.5)$ & $3101(10.7)$ & $2155(8.9)$ & $1364(7.3)$ & $1032(6.2)$ & $936(6.4)$ & -68 & -13.2 \\
\hline Neurosurgery & $1831(5.1)$ & $1573(4.5)$ & $2052(5.6)$ & $2507(7.1)$ & $2108(7.3)$ & $1781(7.3)$ & $1593(8.5)$ & $1511(9.1)$ & $1546(10.5)$ & -16 & -2.1 \\
\hline Radiology and surgery & 31654 (88.6) & $30522(86.8)$ & $31601(86.0)$ & $30293(86.3)$ & $25755(89.0)$ & $21653(89.1)$ & $17119(91.2)$ & $15380(92.2)$ & $13873(94.2)$ & -56 & -9.8 \\
\hline Anesthesiology & $1713(4.8)$ & $1663(4.7)$ & $1559(4.2)$ & $1399(4.0)$ & $850(2.9)$ & $626(2.6)$ & $421(2.2)$ & $358(2.1)$ & $218(1.5)$ & -87 & -22.7 \\
\hline $\begin{array}{l}\text { Interventional pain management } \\
\text { and pain management ( } 09 \text { and } 72)\end{array}$ & $1479(4.1)$ & $2123(6.0)$ & $2635(7.2)$ & $2597(7.4)$ & $1754(6.1)$ & $1552(6.4)$ & $886(4.7)$ & $701(4.2)$ & $462(3.1)$ & -69 & -13.5 \\
\hline PM\&R & $116(0.3)$ & $154(0.4)$ & $257(0.7)$ & $231(0.7)$ & $241(0.8)$ & $222(0.9)$ & $142(0.8)$ & $107(0.6)$ & $86(0.6)$ & -26 & -3.7 \\
\hline Pain management group & $3308(9.3)$ & $3940(11.2)$ & $4451(12.1)$ & $4227(12.0)$ & $2845(9.8)$ & $2400(9.9)$ & $1449(7.7)$ & $1166(7.0)$ & $766(5.2)$ & -77 & -16.7 \\
\hline Other & $754(2.1)$ & $713(2.0)$ & $693(1.9)$ & $571(1.6)$ & $349(1.2)$ & $262(1.1)$ & $196(1.0)$ & $135(0.8)$ & $93(0.6)$ & -88 & -23.0 \\
\hline Total & 35716 & 35175 & 36745 & 35091 & 28949 & 24315 & 18764 & 16681 & 14732 & -59 & -10.5 \\
\hline \multicolumn{12}{|l|}{ Kyphoplasty (n (\%)) } \\
\hline Radiology & $13276(24.3)$ & $17115(27.7)$ & $18584(29.3)$ & $19317(30.7)$ & $17251(29.8)$ & $18822(31.9)$ & $20412(33.4)$ & $20783(33.6)$ & $20265(33.4)$ & 53 & 5.4 \\
\hline Orthopedic surgery & $25398(46.5)$ & $25989(42.1)$ & $25979(41.0)$ & $24364(38.7)$ & $21645(37.4)$ & $20240(34.3)$ & $19879(32.5)$ & $18854(30.5)$ & $18329(30.2)$ & -28 & -4.0 \\
\hline Neurosurgery & $13487(24.7)$ & $15148(24.5)$ & 14939 (23.6) & 14745 (23.4) & $13704(23.7)$ & $14034(23.8)$ & $13608(22.2)$ & $13720(22.2)$ & $13357(22.0)$ & -1 & -0.1 \\
\hline Radiology and surgery & $52161(95.4)$ & $58252(94.3)$ & $59502(93.9)$ & $58426(92.7)$ & $52600(90.9)$ & 53096 (89.9) & $53899(88.1)$ & $53357(86.3)$ & $51951(85.6)$ & 0 & -0.1 \\
\hline Anesthesiology & $923(1.7)$ & $1142(1.8)$ & $1315(2.1)$ & $1558(2.5)$ & $1412(2.4)$ & $1438(2.4)$ & $1920(3.1)$ & $2160(3.5)$ & $2135(3.5)$ & 131 & 11.1 \\
\hline $\begin{array}{l}\text { Interventional pain management } \\
\text { and pain management ( } 09 \text { and } 72)\end{array}$ & $532(1.0)$ & $1233(2.0)$ & $1271(2.0)$ & $1668(2.6)$ & $2461(4.3)$ & $2880(4.9)$ & $3700(6.0)$ & $4552(7.4)$ & $4664(7.7)$ & 777 & 31.2 \\
\hline PM\&R & $134(0.2)$ & $197(0.3)$ & $240(0.4)$ & $484(0.8)$ & $677(1.2)$ & $887(1.5)$ & 860 (1.4) & $1035(1.7)$ & $1218(2.0)$ & 809 & 31.8 \\
\hline Pain management group & $1589(2.9)$ & $2572(4.2)$ & $2826(4.5)$ & $3710(5.9)$ & $4550(7.9)$ & $5205(8.8)$ & 6480 (10.6) & 7747 (12.5) & 8017 (13.2) & 405 & 22.4 \\
\hline Other & $907(1.7)$ & $961(1.6)$ & $1020(1.6)$ & $869(1.4)$ & $740(1.3)$ & $742(1.3)$ & $786(1.3)$ & $735(1.2)$ & $688(1.1)$ & -24 & -3.4 \\
\hline Total & 54657 & 61785 & 63348 & 63005 & 57890 & 59043 & 61165 & 61839 & 60656 & 11 & 1.3 \\
\hline \multicolumn{12}{|l|}{ Combined (n (\%)) } \\
\hline Radiology & $40185(44.5)$ & $42958(44.3)$ & $44370(44.3)$ & $43431(44.3)$ & 37797 (43.5) & 36539 (43.8) & $34574(43.3)$ & $33620(42.8)$ & $31656(42.0)$ & -21 & -2.9 \\
\hline Orthopedic surgery & $28312(31.3)$ & $29095(30.0)$ & $29742(29.7)$ & $28036(28.6)$ & $24746(28.5)$ & $22395(26.9)$ & $21243(26.6)$ & $19886(25.3)$ & $19265(25.6)$ & -32 & -4.7 \\
\hline Neurosurgery & $15318(16.9)$ & $16721(17.2)$ & $16991(17.0)$ & $17252(17.6)$ & $15812(18.2)$ & $15815(19.0)$ & $15201(19.0)$ & $15231(19.4)$ & $14903(19.8)$ & -3 & -0.3 \\
\hline Radiology and surgery & $83815(92.7)$ & $88774(91.6)$ & $91103(91.0)$ & $88719(90.4)$ & $78355(90.2)$ & $74749(89.7)$ & $71018(88.9)$ & $68737(87.5)$ & $65824(87.3)$ & -21 & -3.0 \\
\hline Anesthesiology & $2636(2.9)$ & $2805(2.9)$ & $2874(2.9)$ & $2957(3.0)$ & $2262(2.6)$ & $2064(2.5)$ & $2341(2.9)$ & $2518(3.2)$ & $2353(3.1)$ & -11 & -1.4 \\
\hline $\begin{array}{l}\text { Interventional pain management } \\
\text { and pain management ( } 09 \text { and } 72)\end{array}$ & $2011(2.2)$ & $3356(3.5)$ & $3906(3.9)$ & $4265(4.3)$ & $4215(4.9)$ & $4432(5.3)$ & $4586(5.7)$ & $5253(6.7)$ & $5126(6.8)$ & 155 & 12.4 \\
\hline PM\&R & $250(0.3)$ & $351(0.4)$ & $497(0.5)$ & $715(0.7)$ & $918(1.1)$ & $1109(1.3)$ & $1002(1.3)$ & $1142(1.5)$ & $1304(1.7)$ & 422 & 22.9 \\
\hline Pain management group & $4897(5.4)$ & $65126.7)$ & $7277(7.3)$ & $7937(8.1)$ & $7395(8.5)$ & $7605(9.1)$ & $7929(9.9)$ & $8913(11.4)$ & $8783(11.7)$ & 79 & 7.6 \\
\hline Other & $1661(1.8)$ & $1674(1.7)$ & $1713(1.7)$ & $1440(1.5)$ & $1089(1.3)$ & $1004(1.2)$ & $982(1.2)$ & $870(1.1)$ & $781(1.0)$ & -53 & -9.0 \\
\hline Grand total & 90373 & 96960 & 100093 & 98096 & 86839 & 83358 & 79929 & 78520 & 75388 & -17 & -2.2 \\
\hline
\end{tabular}


Table 4 Percentage of change in volume of vertebroplasty and kyphoplasty procedures by provider specialty in fee for service Medicare beneficiaries

\begin{tabular}{|c|c|c|c|c|c|c|c|}
\hline \multirow[b]{2}{*}{ Physician specialty } & \multicolumn{2}{|l|}{ 2006-2009 } & \multirow{2}{*}{$\begin{array}{l}2009-2010 \\
\text { Change (\%) }\end{array}$} & \multicolumn{2}{|l|}{ 2010-2014 } & \multicolumn{2}{|l|}{$2006-2014$} \\
\hline & Change (\%) & GGR (\%) & & Change (\%) & GGR (\%) & Change (\%) & GGR (\%) \\
\hline \multicolumn{8}{|l|}{ Vertebroplasty } \\
\hline Radiology & -10 & -3.6 & -15 & -45 & -13.7 & -58 & -10.2 \\
\hline Orthopedic surgery & 26 & 8.0 & -16 & -70 & -25.9 & -68 & -13.2 \\
\hline Neurosurgery & 37 & 11.0 & -16 & -27 & -7.5 & -16 & -2.1 \\
\hline Radiology and surgery & -4 & -1.5 & -15 & -46 & -14.3 & -56 & -9.8 \\
\hline Anesthesiology & -18 & -6.5 & -39 & -74 & -28.8 & -87 & -22.7 \\
\hline Pain management & 76 & 20.6 & -32 & -74 & -28.4 & -69 & -13.5 \\
\hline PM\&R & 99 & 25.8 & 4 & -64 & -22.7 & -26 & -3.7 \\
\hline Pain management group & 28 & 8.5 & -33 & -73 & -28.0 & -77 & -16.7 \\
\hline Other & -24 & -8.8 & -39 & -73 & -28.2 & -88 & -23.0 \\
\hline Total & -2 & -0.6 & -18 & -49 & -15.5 & -59 & -10.5 \\
\hline \multicolumn{8}{|l|}{ Kyphoplasty } \\
\hline Radiology & 46 & 13.3 & -11 & 17 & 4.1 & 53 & 5.4 \\
\hline Orthopedic surgery & -4 & -1.4 & -11 & -15 & -4.1 & -28 & -4.0 \\
\hline Neurosurgery & 9 & 3.0 & -7 & -3 & -0.6 & -1 & -0.1 \\
\hline Radiology and surgery & 12 & 3.8 & -10 & -1 & -0.3 & 0 & -0.1 \\
\hline Anesthesiology & 69 & 19.0 & -9 & 51 & 10.9 & 131 & 11.1 \\
\hline Pain management & 214 & 46.3 & 48 & 90 & 17.3 & 777 & 31.2 \\
\hline PM\&R & 261 & 53.4 & 40 & 80 & 15.8 & 809 & 31.8 \\
\hline Pain management group & 133 & 32.6 & 23 & 76 & 15.2 & 405 & 22.4 \\
\hline Other & -4 & -1.4 & -15 & -7 & -1.8 & -24 & -3.4 \\
\hline Total & 15 & 4.8 & -8 & 5 & 1.2 & 11 & 1.3 \\
\hline \multicolumn{8}{|l|}{ Combined } \\
\hline Radiology & 8 & 2.6 & -13 & -16 & -4.3 & -21 & -2.9 \\
\hline Orthopedic surgery & -1 & -0.3 & -12 & -22 & -6.1 & -32 & -4.7 \\
\hline Neurosurgery & 13 & 4.0 & -8 & -6 & -1.5 & -3 & -0.3 \\
\hline Radiology and surgery & 6 & 1.9 & -12 & -16 & -4.3 & -21 & -3.0 \\
\hline Anesthesiology & 12 & 3.9 & -24 & 4 & 1.0 & -11 & -1.4 \\
\hline Pain management & 112 & 28.4 & -1 & 22 & 5.0 & 155 & 12.4 \\
\hline$P M \& R$ & 186 & 41.9 & 28 & 42 & 9.2 & 422 & 22.9 \\
\hline Pain management group & 62 & 17.4 & -7 & 19 & 4.4 & 79 & 7.6 \\
\hline Other & -13 & -4.6 & -24 & -28 & -8.0 & -53 & -9.0 \\
\hline Grand total & 9 & 2.8 & -11 & -13 & -3.5 & -17 & -2.2 \\
\hline
\end{tabular}

GGR, geometric growth rate; PM\&R, physical medicine and rehabilitation.

management (300 patients) in The Lancet (impact factor 30.8 in $2009)^{26}$ which reported a significant benefit for kyphoplasty that was largely unnoticed by the media.

The subsequent publication of multiple randomized controlled trials demonstrating significant benefit for vertebroplasty and/or kyphoplasty has not counterbalanced the earlier NEJM publications. Since 2009, a total of 1187 patients have been included in 8 randomized controlled trials of vertebroplasty and/or kyphoplasty that showed significant benefit of vertebral augmentation. ${ }^{14-21}$ However, these studies were published in journals with a wide range of significantly lower impact factors (as low as 1.31$)^{26}$ at the time of their publication, and thus were largely unnoticed by the media. In addition, all kyphoplasty randomized controlled trials to date have been positive, in contrast with vertebroplasty. In this context, it is not surprising that rates of vertebroplasty declined significantly without a similar decline in kyphoplasty.

Importantly, there is no significant difference in reduction of pain and disability between vertebroplasty and kyphoplasty, which are the primary goals of vertebral augmentation. In a meta-analysis of prospective comparative studies of vertebroplasty and kyphoplasty, Chang et al ${ }^{27}$ included 1429 patients from 20 studies and found that there was no difference in the reduction of pain and disability between the two techniques. Similar findings were reported in another meta-analysis of 845 patients. ${ }^{28}$ A recent systematic review of both vertebroplasty or kyphoplasty for cancer related vertebral compression fractures by Ontario Health Technology concluded that both vertebroplasty and kyphoplasty significantly and rapidly reduced pain intensity in cancer patients with vertebral compression fractures. ${ }^{29}$ These findings are not unexpected as kyphoplasty is essentially an extension of the vertebroplasty procedure-that is, with an additional step of inflation of a balloon tamp within the vertebra prior to cement injection. Nonetheless, the results also reveal that while radiologists are performing less vertebroplasties, they are performing a higher percentage of kyphoplasties compared with earlier time periods.

Vertebral augmentation has gone through several elements of a complex life cycle. In the 1990 s, early US based neurointerventional investigators started performing percutaneous vertebroplasty at the University of Virginia. ${ }^{30}$ The regional/national experience at that time largely grew out of providers that had a unique interaction with that pioneering group. In 1997, when their seminal article on augmentation was published, ${ }^{30}$ interest 
Table 5 Vertebroplasty and kyphoplasty services by place of services for fee for service Medicare beneficiaries from 2008 to 2014

\begin{tabular}{|c|c|c|c|c|c|c|c|c|c|}
\hline PLCR & F2008 & F2009 & F2010 & F2011 & F2012 & F2013 & F2014 & Change (\%) & GGR (\%) \\
\hline \multicolumn{10}{|c|}{ Vertebroplasty (n (\%)) } \\
\hline ASC & $1342(3.7)$ & $1489(4.2)$ & 1043 (3.6) & $642(2.6)$ & $534(2.8)$ & 438 (2.6) & $347(2.4)$ & -74.1 & -20.2 \\
\hline Outpatient & $16266(44.3)$ & $15865(45.2)$ & $13430(46.4)$ & 11534 (47.4) & $10095(53.8)$ & 9585 (57.5) & 8479 (57.6) & -47.9 & -10.3 \\
\hline Inpatient & $14296(38.9)$ & $13184(37.6)$ & $10804(37.3)$ & $8753(36.0)$ & 6737 (35.9) & 5813 (34.8) & 5247 (35.6) & -63.3 & -15.4 \\
\hline Office & $4841(13.2)$ & $4553(13.0)$ & $3672(12.7)$ & $3386(13.9)$ & $1398(7.4)$ & $845(5.1)$ & $659(4.5)$ & -86.4 & -28.3 \\
\hline Total & 36745 & 35091 & 28949 & 24315 & 18764 & 16681 & 14732 & -59.9 & -14.1 \\
\hline \multicolumn{10}{|c|}{ Kyphoplasty n (\%)) } \\
\hline ASC & $283(0.4)$ & 909 (1.4) & $1721(3.0)$ & $2322(3.9)$ & $1953(3.2)$ & $2205(3.6)$ & $2192(3.6)$ & 674.6 & 40.7 \\
\hline Outpatient & $24084(38.0)$ & $29666(47.1)$ & $28749(49.7)$ & $31465(53.3)$ & 32264 (52.7) & $32185(52.0)$ & $31357(51.7)$ & 30.2 & 4.5 \\
\hline Inpatient & $38862(61.3)$ & $32296(51.3)$ & $27224(47.0)$ & $25190(42.7)$ & $22960(37.5)$ & $20904(33.8)$ & $19300(31.8)$ & -50.3 & -11.0 \\
\hline Office & $119(0.2)$ & $134(0.2)$ & $196(0.3)$ & $66(0.1)$ & $3988(6.5)$ & 6545 (10.6) & 7807 (12.9) & 6460.5 & 100.9 \\
\hline Total & 63348 & 63005 & 57890 & 59043 & 61165 & 61839 & 60656 & -4.2 & -0.7 \\
\hline \multicolumn{10}{|c|}{ Combined n (\%)) } \\
\hline ASC & 1625 (1.6) & 2398 (2.4) & 2764 (3.2) & 2964 (3.6) & 2487 (3.1) & $2643(3.4)$ & $2539(3.4)$ & 56.2 & 7.7 \\
\hline Outpatient & $40350(40.3)$ & 45531 (46.4) & $42179(48.6)$ & 42999 (51.6) & $42359(53.0)$ & $41770(53.2)$ & $39836(52.8)$ & -1.3 & -0.2 \\
\hline Inpatient & 53158 (53.1) & $45480(46.4)$ & $38028(43.8)$ & $33943(40.7)$ & 29697 (37.2) & $26717(34.0)$ & 24547 (32.6) & -53.8 & -12.1 \\
\hline Office & $4960(5.0)$ & $4687(4.8)$ & $3868(4.5)$ & $3452(4.1)$ & $5386(6.7)$ & 7390 (9.4) & $8466(11.2)$ & 70.7 & 9.3 \\
\hline Total & 100093 & 98096 & 86839 & 83358 & 79929 & 78520 & 75388 & -24.7 & -4.6 \\
\hline
\end{tabular}

further increased and early generation vertebroplasty kits were soon developed. Over the next 20 years, multiple cohort studies and systematic reviews of these studies supported the use of vertebral augmentation. ${ }^{6} 73132$ and multiple professional medical societies supported their use. ${ }^{33}$ In 2009 , the NEJM trials were published which showed lack of effectiveness of vertebroplasty compared with placebo. ${ }^{9}{ }^{10}$ However, limitations of these trials included the inclusion of fractures up to 12 months old, and periosteal infiltration of local anesthetic, an active control, that may have resolved pain that primarily arose from the adjacent structures in more chronic fractures. ${ }^{34} 35$

Since the NEJM trials were published, there have been multiple additional randomized controlled trials supporting the use of both vertebroplasty and kyphoplasty for osteoporotic and cancer related fractures. ${ }^{14-21}$ Meta-analyses including the NEJM trials were also supportive of vertebral augmentation. ${ }^{36}{ }^{37}$ These led to multiple international society guidelines supporting the use of vertebral augmentation, ${ }^{38-40}$ and endorsement of vertebroplasty for both osteoporotic and cancer related fractures by the National Institute for Health and Care Excellence that advises the National Health Service of England and Wales. ${ }^{41} 42$ Most recently, the Vertebroplasty for Acute Painful Osteoporotic fractURes (VAPOUR) trial was published in The Lancet. ${ }^{21}$ VAPOUR was a multicenter randomized controlled trial designed to assess the efficacy of vertebroplasty for osteoporotic fractures in a subgroup of patients with recent ( $<6$ weeks) onset severe pain. Notably, the placebo procedure was subcutaneous local anesthetic infiltration, closer to conservative medical management. This trial showed strong benefit to performing vertebroplasty, and also demonstrated that conservative medical therapy is not without risk; two medically managed patients developed spinal cord compression from further collapse of their fractures. ${ }^{2143}$ The effects of this trial on procedure utilization rates will become evident in the next few years.

There are many limitations to our analysis. Firstly, we reviewed the CMS database, which excludes Medicare Advantage patients. In addition, there are private payer insurers that reimburse for this procedure that are by definition not included in this analysis. In addition, there may have been miscoding of procedures, and other limitations to using claims based data. ${ }^{44}$ Nonetheless, Medicare is the largest health care payer in the USA for vertebral augmentation, providing a reliable dataset to evaluate procedure utilization.

\section{CONCLUSION}

There was an overall decline in vertebroplasty and kyphoplasty procedures in the FFS Medicare population between 2004 and 2014, and particularly since 2009. Our analysis suggests that the NEJM publications have had a considerable long term impact on utilization of vertebral augmentation procedures in the Medicare population. We look forward to assessing the impact of the recently published VAPOUR trial in future analyses.

Contributors JAH and LM constructed the study, analyzed/synthesized the data, and crafted the original manuscript. VP organized the data. RVC, ALB, and JDB edited the preliminary draft and provided meaningful editorial suggestions. All authors take responsibility for the final manuscript.

Competing interests JAH has an ongoing consulting relationship with Medtronic and in the past 36 months consulted for Carefusion. Both companies make products for vertebral augmentation. JB: shareholder in Medtronic and Stryker; research support from Merit/DFine and Medtronic.

Provenance and peer review Not commissioned; internally peer reviewed.

\section{REFERENCES}

1 Melton LJ III, Kallmes DF. Epidemiology of vertebral fractures: implications for vertebral augmentation. Acad Radiol 2006;13:538-45.

2 Rostom S, Allali F, Bennani L, et al. The prevalence of vertebral fractures and health-related quality of life in postmenopausal women. Rheumatol Int 2012;32:971-80

3 Baaj AA, Downes $K$, Vaccaro AR, et al. Trends in the treatment of lumbar spine fractures in the United States: a socioeconomics perspective: clinical article. I Neurosur Spine 2011;15:367-70.

4 Hulme PA, Krebs J, Ferguson SJ, et al. Vertebroplasty and kyphoplasty: a systematic review of 69 clinical studies. Spine 2006;31:1983-2001.

5 Taylor RS, Taylor RJ, Fritzell P. Balloon kyphoplasty and vertebroplasty for vertebral compression fractures: a comparative systematic review of efficacy and safety. Spine 2006;31:2747-55.

6 Bouza C, López T, Magro A, et al. Efficacy and safety of balloon kyphoplasty in the treatment of vertebral compression fractures: a systematic review. Euro Spine J 2006;15:1050-67.

7 Hochmuth K, Proschek D, Schwarz W, et al. Percutaneous vertebroplasty in the therapy of osteoporotic vertebral compression fractures: a critical review. Euro Rad 2006;16:998-1004. 
8 Manchikanti L, Pampati V, Hirsch JA. Analysis of utilization patterns of vertebroplasty and kyphoplasty in the Medicare population. J Neurointerv Surgery 2013;5:467-72.

9 Buchbinder R, Osborne RH, Ebeling PR, et al. A randomized trial of vertebroplasty for painful osteoporotic vertebral fractures. N Engl J Med 2009;361:557-68.

10 Kallmes DF, Comstock BA, Heagerty PJ, et al. A randomized trial of vertebroplasty for osteoporotic spinal fractures. N Engl J Med 2009;361:569-79.

11 Manchikanti L, Falco FJ, Singh V, et al. Utilization of interventional techniques in managing chronic pain in the Medicare population: analysis of growth patterns from 2000 to 2011. Pain Physician 2012;15:E969-82.

12 Manchikanti L, Pampati V, Falco FJ, et al. An updated assessment of utilization of interventional pain management techniques in the Medicare population: 2000 2013. Pain Physician 2015;18:E115-27.

13 Manchikanti L, Pampati V, Hirsch JA. Utilization of interventional techniques in managing chronic pain in Medicare population from 2000 to 2014: an analysis of patterns of utilization. Pain Physician 2016;19:E531-46.

14 Berenson J, Pflugmacher R, Jarzem P, et al. Balloon kyphoplasty versus non-surgical fracture management for treatment of painful vertebral body compression fractures in patients with cancer: a multicentre, randomised controlled trial. Lancet Oncol 2011;12:225-35.

15 Blasco J, Martinez-Ferrer A, Macho J, et al. Effect of vertebroplasty on pain relief, quality of life, and the incidence of new vertebral fractures: a 12-month randomized follow-up, controlled trial. J Bone Mineral Res 2012;27:1159-66.

16 Farrokhi MR, Alibai E, Maghami Z. Randomized controlled trial of percutaneous vertebroplasty versus optimal medical management for the relief of pain and disability in acute osteoporotic vertebral compression fractures. J Neurosurg Spine 2011;14:561-9.

17 Klazen CA, Lohle PN, de Vries J, et al. Vertebroplasty versus conservative treatment in acute osteoporotic vertebral compression fractures (Vertos II): an open-label randomised trial. Lancet 2010;376:1085-92.

18 Wardlaw D, Cummings SR, Van Meirhaeghe J, et al. Efficacy and safety of balloon kyphoplasty compared with non-surgical care for vertebral compression fracture (FREE): a randomised controlled trial. Lancet 2009;373:1016-24.

19 Chen D, An ZQ, Song S, et al. Percutaneous vertebroplasty compared with conservative treatment in patients with chronic painful osteoporotic spinal fractures. J Clin Neurosci 2014;21:473-7.

20 Yang EZ, Xu JG, Huang GZ, et al. Percutaneous vertebroplasty versus conservative treatment in aged patients with acute osteoporotic vertebral compression fractures: a prospective randomized controlled clinical study. Spine 2016;41:653-60.

21 Clark W, Bird P, Gonski P, et al. Safety and efficacy of vertebroplasty for acute painful osteoporotic fractures (VAPOUR): a multicentre, randomised, double-blind, placebo-controlled trial. Lancet 2016;388:1408-16.

22 Vandenbroucke JP, Von Elm E, Altman DG, et al. [Strengthening the reporting of observational studies in epidemiology (STROBE): explanation and elaboration]. Gac Sanit 2009;23:158.

23 Specialty utilization data files from CMS. http://www.cms.hhs.gov/

24 Cox M, Levin DC, Parker L, et al. Vertebral augmentation after recent randomized controlled trials: a new rise in kyphoplasty volumes. J Am Coll Radiol 2016;13:28-32.

25 Long SS, Morrison WB, Parker L. Vertebroplasty and kyphoplasty in the United States: provider distribution and guidance method, 2001-2010. Am J Roentgenol 2012;199:1358-64.

26 International Scientific Institute. Journal Impact Factor List. http://www.scijournal.org

27 Chang X, Lv YF, Chen B, et al. Vertebroplasty versus kyphoplasty in osteoporotic vertebral compression fracture: a meta-analysis of prospective comparative studies. Int Orthop 2015;39:491-500.
28 Wang H, Sribastav SS, Ye F, et al. Comparison of percutaneous vertebroplasty and balloon kyphoplasty for the treatment of single level vertebral compression fractures: a meta-analysis of the literature. Pain Physician 2015;18:209-22.

29 Health Quality Onatario. Vertebral augmentation involving vertebroplasty or kyphoplasty for cancer-related vertebral compression fractures: a systematic review. Ont Health Technol Assess Ser 2016;16:1-202.

30 Jensen ME, Evans AJ, Mathis JM, et al. Percutaneous polymethylmethacrylate vertebroplasty in the treatment of osteoporotic vertebral body compression fractures: technical aspects. AJNR Am J Neuroradiol 1997;18:1897-904.

31 Anselmetti GC, Manca A, Hirsch J, et al. Percutaneous vertebroplasty in osteoporotic patients: an institutional experience of 1,634 patients with long-term follow-up. J Vasc Interv Radiol 2011;22:1714-20.

32 Jha RM, Yoo AJ, Hirsch AE, et al. Predictors of successful palliation of compression fractures with vertebral augmentation: single-center experience of 525 cases. J Vasc Interv Radiol 2009;20:760-8.

33 Jensen ME, McGraw JK, Cardella JF, et al. Position statement on percutaneous vertebral augmentation: a consensus statement developed by the American Society of Interventional and Therapeutic Neuroradiology, Society of Interventional Radiology, American Association of Neurological Surgeons/Congress of Neurological Surgeons, and American Society of Spine Radiology. AJNR Am J Neuroradiol 2007;28:1439-43.

34 Manchikanti L, Giordano J, Fellows B, et al. Placebo and nocebo in interventional pain management: a friend or a foe_or simply foes? Pain Physician 2011;14:E157-75.

35 Chandra RV, Yoo AJ, Hirsch JA. Vertebral augmentation: update on safety, efficacy, cost effectiveness and increased survival? Pain Physician 2013;16:309-20.

36 Anderson PA, Froyshteter AB, Tontz WL Jr. Meta-analysis of vertebral augmentation compared to conservative treatment for osteoporotic spinal fractures. J Bone Mineral Res 2013;28:372-82.

37 Shi MM, Cai XZ, Lin T, et al. Is there really no benefit of vertebroplasty for osteoporotic vertebral fractures? A meta-analysis. Clin Orthop Relat Res 2012;470:2785-99.

38 Barr JD, Jensen ME, Hirsch JA, et al. Position statement on percutaneous vertebral augmentation: a consensus statement developed by the Society of Interventional Radiology (SIR), American Association of Neurological Surgeons (AANS) and the Congress of Neurological Surgeons (CNS), American College of Radiology (ACR), American Society of Neuroradiology (ASNR), American Society of Spine Radiology (ASSR), Canadian Interventional Radiology Association (CIRA), and the Society of Neurolnterventional Surgery (SNIS). J Vasc Interv Radiol 2014;25:171-81.

39 Chandra RV, Meyers PM, Hirsch JA, et al. Vertebral augmentation: report of the Standards and Guidelines Committee of the Society of Neurolnterventional Surgery. Neurointerv Surg 2014;6:7-15.

40 Terpos E, Morgan G, Dimopoulos MA, et al. International Myeloma Working Group recommendations for the treatment of multiple myeloma-related bone disease. J Clin Oncol 2013;31:2347-57.

41 National Institute for Health and Clinical Excellence. NICE technology appraisal guidance TA279. Percutaneous vertebroplasty and percutaneous balloon kyphoplasty for treating osteoporotic vertebral compression fractures. April 2013. https://www. nice.org.uk/guidance/ta279

42 National Institute for Health and Clinical Excellence. Metastatic spinal cord compression: Diagnosis and management of patients at risk of or with metastatic spinal cord compression. NICE Clinical guideline 75 November 2008. https://www.nice.org.uk/ guidance/cg75/chapter/1-Guidance\#treatment-of-spinal-metastases-and-mscc

43 Hirsch JA, Chandra RV. Resurrection of evidence for vertebroplasty? Lancet. 2016;388:1356-7.

44 McCullough BJ, Comstock BA, Deyo RA, et al. Major medical outcomes with spinal augmentation vs conservative therapy. JAMA Intern Med 2013;173:1514-21. 